\title{
Comparative Study of Coupling Factors for Assessment of Low-Frequency Magnetic Field Exposure
}

\author{
Jae-Hoon Shim ${ }^{1}$, Min-Soo Choi ${ }^{1}$, Kyu-Jin Jung ${ }^{1}$, Jong-Hwa Kwon ${ }^{2}$, and Jin-Kyu Byun ${ }^{1 *}$ \\ ${ }^{1}$ Department of Electrical Engineering, Soongsil University, Seoul 156-743, Korea \\ ${ }^{2}$ Radio Technology Research Department, Electronics and Telecommunications Research Institute, Daejeon 305-700, Korea
}

(Received 21 October 2016, Received in final form 17 November 2016, Accepted 18 November 2016)

\begin{abstract}
In this paper, coupling factors are calculated based on numerical analysis in order to assess various nonuniform low-frequency magnetic field exposure situations. Two types of non-uniform magnetic field sources are considered; circular coil and parallel wires with balanced currents. For each magnetic field source, source current values are determined so that reference magnetic field magnitude can be measured at the specified point on the human model. Various exposure situations are investigated by changing parameters such as the distance between source and human model, radius of circular coil, and the gap between parallel wires. For equivalent human models, prolate spheroid model and simplified human model from IEC 62311 standard are used. The calculated coupling factor values are compared with those obtained by $2 \mathrm{D}$ uniform disk human model, and the dependence of coupling factor on the choice of equivalent human model is analyzed.
\end{abstract}

Keywords : coupling factor, human model, magnetic field exposure, non-uniform field

\section{Introduction}

With increasing number of devices in everyday life that utilize electric and magnetic fields (EMFs), such as mobile phones, induction cookers and wireless chargers, the public concern about exposure to EMFs remains high. To protect general public from exposure to EMFs, various safety guidelines have been established [1-3]. EMF safety guidelines such as International Commission on NonIonizing Radiation Protection (ICNIRP) 2010 guideline and IEEE c95.1-2005 standard define exposure limits in terms of two different categories of physical quantities, i.e., basic restrictions and reference level. The basic restrictions are based on established health effects and include induced current density or induced electric field inside the human body. Since basic restrictions are difficult to measure directly, reference levels are provided for practical exposure assessment. Reference levels include electric and magnetic field strength measured in free space where human body can be located.

Reference levels are derived from the basic restrictions using computational techniques assuming uniform field

CThe Korean Magnetics Society. All rights reserved.

*Corresponding author: Tel: +82-2-820-0644

Fax: +82-2-817-7961, e-mail: jkbyun@ssu.ac.kr exposure for conservative estimation. However, this leads to the overestimation of the induced current density for practical situations when magnetic field is not uniform. In order to alleviate this problem, coupling factor $K$ was introduced that enable exposure assessment for complex non-uniform magnetic field exposure situations. In IEC 62226-2-1 standard, coupling factor $K$ is calculated for various non-uniform magnetic field sources including circular coils and balanced parallel wires [4].

These coupling factors can greatly facilitate the procedures for magnetic field exposure assessment. However, the value of the coupling factor $K$ can vary depending on the human body model used in the simulation. In the IEC standards, the coupling factor $K$ has been calculated using a simple equivalent 2D human model (uniform disk) [4]. Since actual magnetic field exposure of human body happens in 3D environment, there are some exposure situations where a simple 2D model cannot be used at all. Also, over-simplified geometry of the human model such as uniform disk can introduce some discrepancies in the value of the calculated coupling factor. However, there have been few works to improve accuracy of coupling factor calculations using 3D human models for lowfrequency magnetic field exposure situation.

In this paper, coupling factor $K$ is calculated using two separate 3D equivalent human models (prolate spheroid 
and simplified human model from IEC 62311 standard) for various non-uniform magnetic field sources (circular coils and parallel wires). The calculated $K$ values are compared with those given in the IEC 62226-2-1 standard, and dependence of coupling factor $K$ on the choice of the equivalent human model is analyzed.

\section{Coupling Factor and Modeling of Non-uniform Magnetic Field Source}

\subsection{Definition and meaning of coupling factor $K$}

The definition of coupling factor $K$ is given in IEC 62226-2-1 standard as follows:

$$
K=\frac{J_{\text {nonuniform }}}{J_{\text {uniform }}}
$$

where $J_{\text {nonuniform }}$ is the maximum induced current density in the human model exposed to the non-uniform magnetic field from a localized source, and $J_{\text {uniform }}$ is the maximum induced current density in the human model exposed to the uniform magnetic field. Assuming that strength of the non-uniform magnetic field is the same as the uniform field at the model surface location closest to the source, the current density induced by a non-uniform field $\left(J_{\text {nonuniform}}\right)$ is always lower than that by a uniform field $\left(J_{\text {uniform}}\right)$ [4]. Hence, the coupling factor $K$ quantifies the reduction of induced current for non-uniform field, and the range of $K$ is given as $0<K<1$. If coupling factor $K$ is known for specific non-uniform magnetic field exposure situation, the field strength measured at the free space can be multiplied by $K$ and compared with reference levels to determine compliance with EMF safety guidelines.

\subsection{Equivalent human models}

Figure 1 shows homogeneous prolate spheroid and

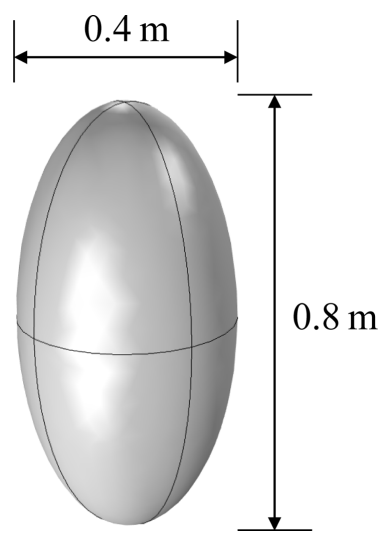

Fig. 1. (Color online) Prolate spheroid model for coupling factor calculation.

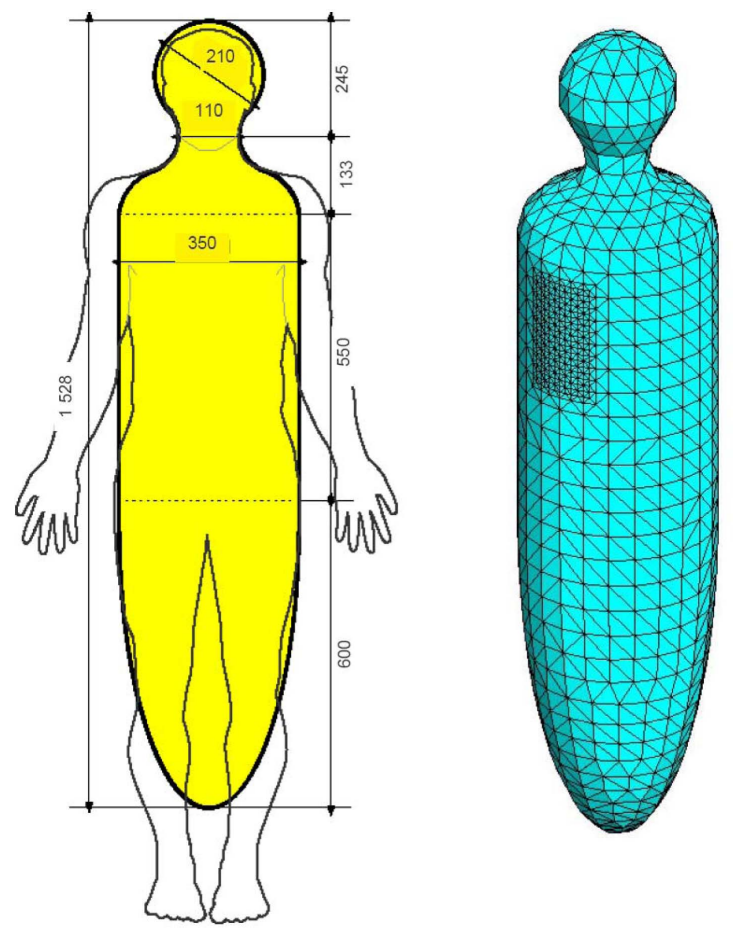

Fig. 2. (Color online) Simplified human model from IEC 62311 for coupling factor calculation [5].

simplified human model used for coupling factor calculation. The height and width of the prolate spheroid is 0.8 $\mathrm{m}$ and $0.4 \mathrm{~m}$, respectively. Figure 2 shows simplified human model from IEC 62311 standard [5]. The model is composed of head and torso, and the height of the model is $152.8 \mathrm{~cm}$. The electrical conductivity is set as $\sigma=0.2$ $\mathrm{S} / \mathrm{m}$ for both models.

\subsection{Calculation of current for specified magnetic field} intensity: circular coil

For calculation of coupling factor $K$ using (1), induced current density from non-uniform magnetic field $J_{\text {nonuniform }}$ should be calculated when the magnetic field at the point of the human model closest to the localized source has the same magnitude as that of the uniform field exposure case. Hence, for a localized magnetic field source, it is necessary to set the source current so that the specified magnetic field magnitude can be obtained at a specific point.

Two types of non-uniform magnetic field sources are investigated in this paper; circular coil and two balanced parallel wires. In this section, the source current is calculated for circular coil that will produce $1.25 \mu \mathrm{T}$ at the specified distance from the coil. The value of $1.25 \mu \mathrm{T}$ was chosen according to IEC 62226-2-1 standard for comparison of calculated $K$ values with those in the 


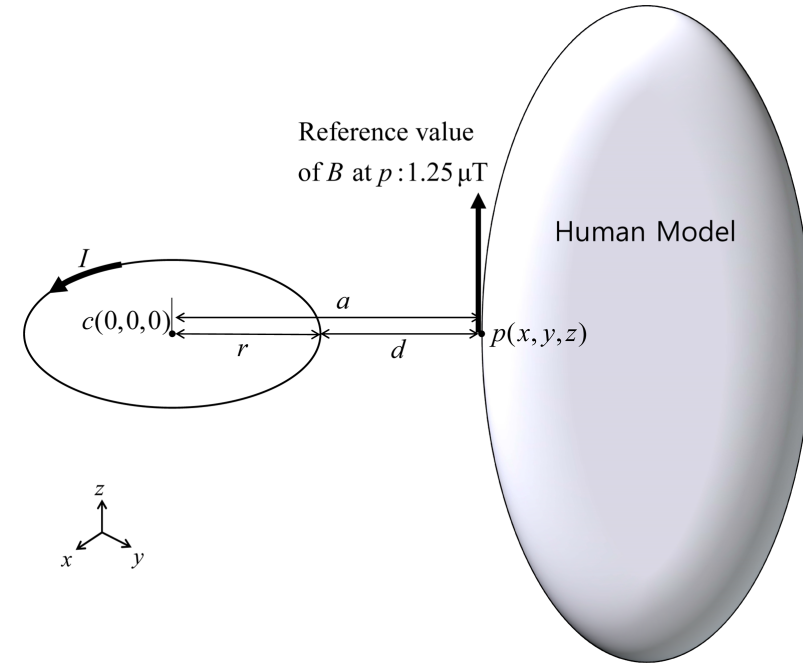

Fig. 3. (Color online) Circular coil and the point of magnetic field calculation $p$ on human model.

standard in chapter 3 [4]. Figure 3 shows the circular coil and the point of magnetic field calculation on the human model. Assuming line current source with center at origin, the magnetic field at a specific point $p(x, y, z)$ can be written as [4]:

$$
\begin{aligned}
& H_{r}=\frac{I k z}{4 \pi a \sqrt{a r}}\left(-F(k)+\frac{r^{2}+a^{2}+z^{2}}{(r-a)^{2}+z^{2}} E(k)\right) \\
& H_{z}=\frac{I k}{4 \pi \sqrt{a r}}\left(F(k)+\frac{r^{2}-a^{2}-z^{2}}{(r-a)^{2}+z^{2}} E(k)\right) \\
& k=\sqrt{\frac{4 a r}{(r+a)^{2}+z^{2}}}, a=\sqrt{x^{2}+y^{2}} \\
& F(k)=\int_{0}^{\pi / 2} \frac{1}{\sqrt{\left(1-k^{2} \sin ^{2} \theta\right)}} d \theta \\
& E(k)=\int_{0}^{\pi / 2} \sqrt{\left(1-k^{2} \sin ^{2} \theta\right)} d \theta
\end{aligned}
$$

where $r$ is the coil radius, $H_{r}$ and $H_{z}$ are radial and vertical magnetic field, respectively, and $F(k)$ and $E(k)$ are elliptical integrals of 1st and 2nd order, respectively.

When the point $p(x, y, z)$ is on the $x-y$ plane, $z=0$ and $H_{r}=0$ from (2). Thus corresponding source current $I$ for specified magnetic field strength $H$ at point $p$ can be derived from (3) as:

$$
I=\frac{4 \pi H \sqrt{a r}(r-a)}{k[(r-a) K(k)+(r+a) E(k)]} .
$$

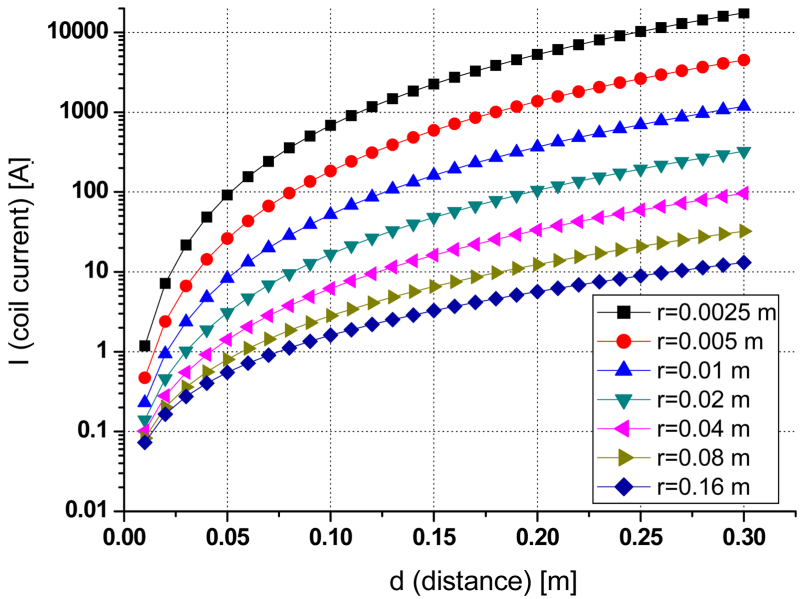

Fig. 4. (Color online) Circular coil current $I$ to produce 1.25 $\mu \mathrm{T}$ at distance $d$ for various coil radius $r$ (log scale).

Using (6), circular coil current $I$ to produce $1.25 \mu \mathrm{T}$ at $p$ is plotted for various values of coil radius $r$ and distance $d$ $=a-r$ between coil and point $p$ (Fig. 4). It is observed that the coil current $I$ is increased as distance $d$ is increased and coil radius $r$ is decreased.

\subsection{Calculation of current for specified magnetic field intensity: parallel wires}

In this section, the source current is calculated for 2 parallel wires that will produce $1.25 \mu \mathrm{T}$ at the specified distance from the wire. Figure 5 shows 2 parallel wires with balanced currents and the point of magnetic field calculation on the human model. Assuming line current

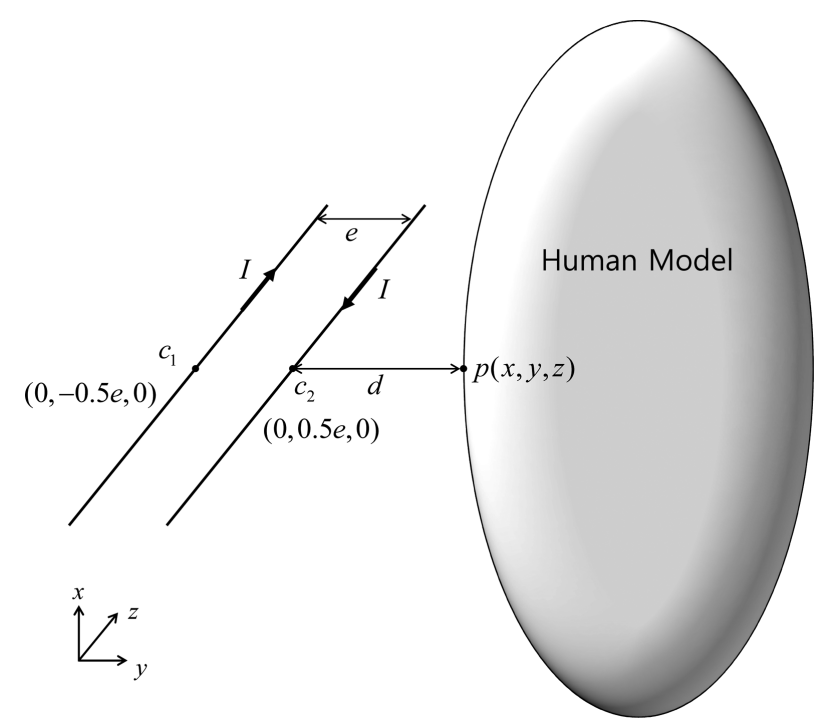

Fig. 5. (Color online) Two parallel wires with balanced currents and the point of magnetic field calculation $p$ on human model. 
source with centers at $c_{1}(0,-0.5 e, 0)$ and $c_{2}(0,-0.5 e, 0)$, the magnetic field at a specific point $p(x, y, z)$ can be written as [4]:

$$
\begin{aligned}
& H_{x}=\frac{-I}{2 \pi}\left[\frac{\frac{e}{2}-y}{\left(x^{2}+\left(y-\frac{e}{2}\right)^{2}\right)}+\frac{\frac{e}{2}+y}{\left(x^{2}+\left(y+\frac{e}{2}\right)^{2}\right)}\right] \\
& H_{y}=\frac{-I}{2 \pi}\left[\frac{x}{\left(x^{2}+\left(y-\frac{e}{2}\right)^{2}\right)}-\frac{x}{\left(x^{2}+\left(y+\frac{e}{2}\right)^{2}\right)}\right]
\end{aligned}
$$

where $e$ is the gap between the 2 wires, and $H_{x}$ and $H_{y}$ are $x$ and $y$ component of magnetic field, respectively.

When the point $p(x, y, z)$ is on the $y$-z plane, $x=0$ and $H_{y}=0$ from (8). Thus corresponding source current $I$ for specified magnetic field strength $H$ at point $p$ can be derived from (7) as:

$$
I=\frac{2 \pi H d(d+e)}{e}
$$

where $d=y-0.5 e$ is the distance between human model and a wire closer to it. Using (9), parallel wire current $I$ to produce $1.25 \mu \mathrm{T}$ at $p$ is plotted for various values of $d$ and gap $e$ between two wires (Fig. 6). It is observed that the wire current $I$ is increased as $d$ is increased and $e$ is decreased.

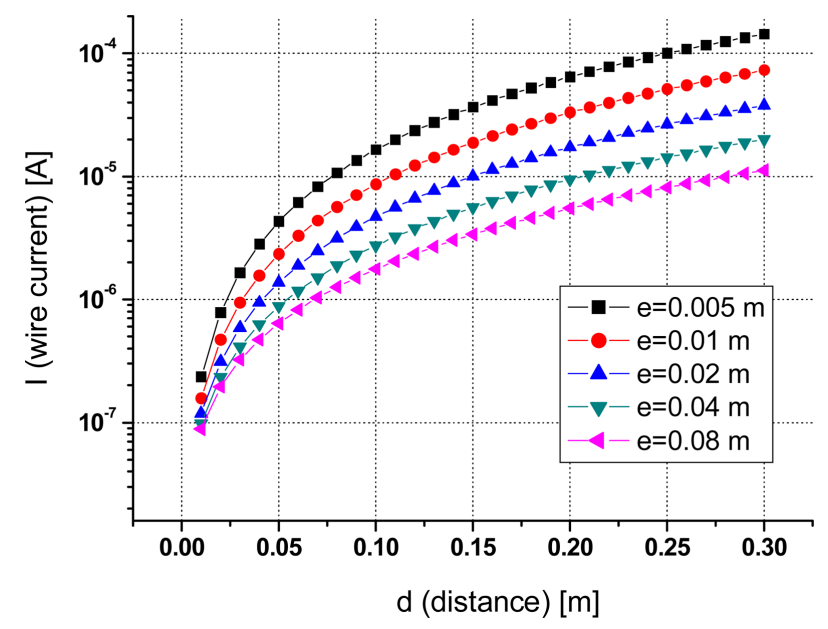

Fig. 6. (Color online) Parallel wire current $I$ to produce 1.25 $\mu \mathrm{T}$ at distance $d$ between human model and wire for various values of $e$ (gap between wires) (log scale).

\section{Calculation and Analysis of Coupling Factor}

\subsection{Exposure situations for coupling factor calcula-} tion

In this chapter, the coupling factors are calculated for 2 different types of non-uniform magnetic field sources; circular coil and parallel wires. For each magnetic field source, 2 different types of equivalent human models are used; prolate spheroid model and IEC 62311 human model described in section 2.2. For prolate spheroid, the exposure situations are described in Figs. 3 and 5. For IEC 62311 model, the exposure situations are depicted in Figs. 7 and 8.

The frequency of coil current $I$ is $50 \mathrm{~Hz}$, and source current is set to obtain reference magnetic flux density of $1.25 \mu \mathrm{T}$ at the specified point $p$ as was calculated in section 2.3 and 2.4. The range of distance $d$ between source and human model is $1-30 \mathrm{~cm}$. For circular coil, the range of coil radius $r$ is $0.25-16 \mathrm{~cm}$. For parallel wires, the range of gap $e$ between wires is $0.5-8 \mathrm{~cm}$.

\subsection{Calculation and analysis of coupling factor $K$}

In order to calculate coupling factor $K$ using (1), both $J_{\text {uniform }}$ (maximum induced current density for uniform field exposure) and $J_{\text {nonuniform }}$ (maximum induced current density for non-uniform field exposure) are needed. $J_{\text {nonuniform }}$ is calculated by 3D finite element method using COMSOL Multiphysics software [6]. 99th percentile value

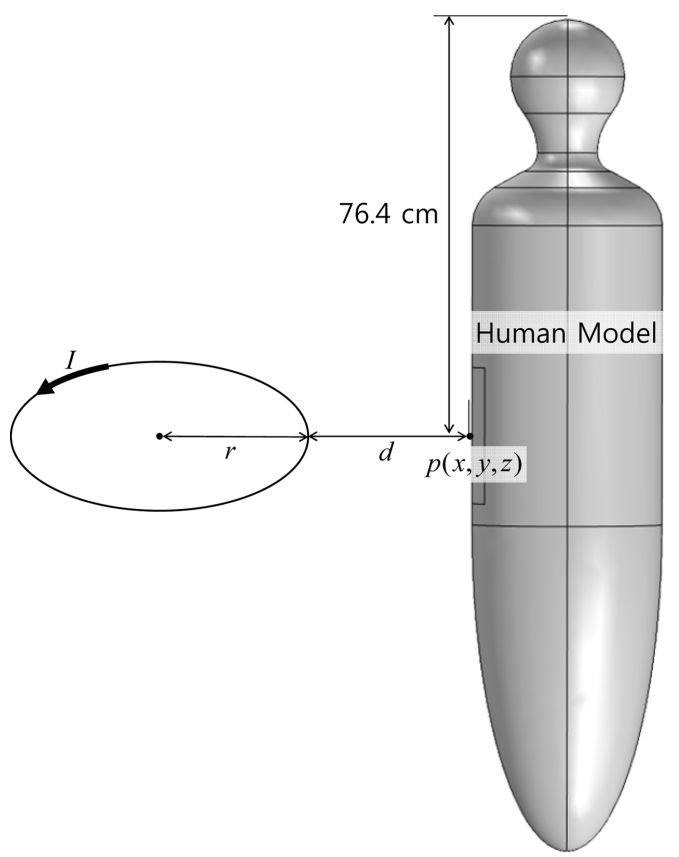

Fig. 7. (Color online) Circular coil exposure situation using IEC 62311 human model. 


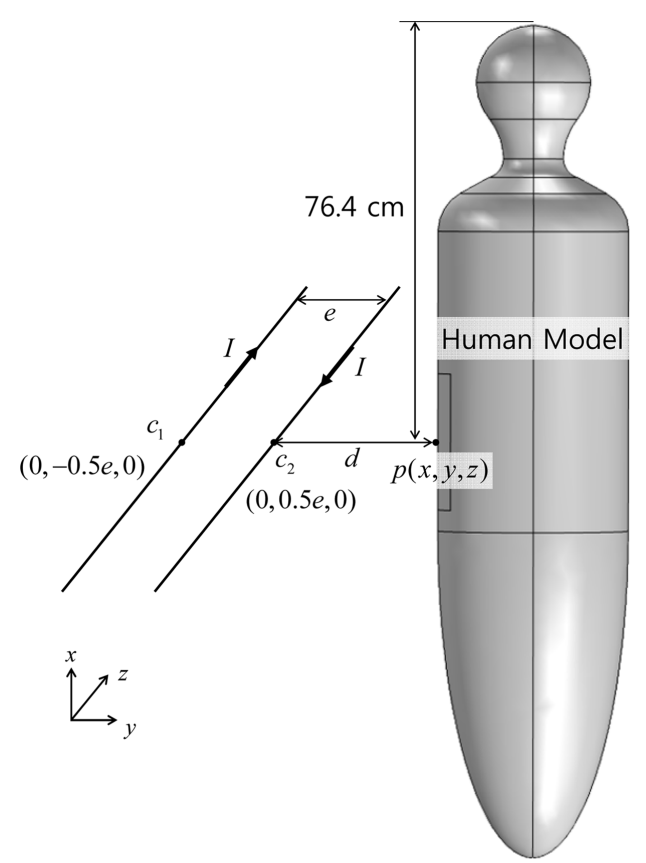

Fig. 8. (Color online) Parallel wires exposure situation using IEC 62311 human model.

of induced current density is used instead of maximum value in order to alleviate numerical errors [7].

As for $J_{\text {uniform}}$, it can be obtained from numerical analysis using uniform field exposure, or it can be analytically calculated under specific conditions. Assuming that the human model is homogeneous body of revolution and the direction of the uniform magnetic field is parallel to the axis of revolution, $J_{\text {uniform }}$ can be derived from Faraday's law as follows:

$$
J_{\text {uniform }}=\sigma \pi f B r_{h}
$$

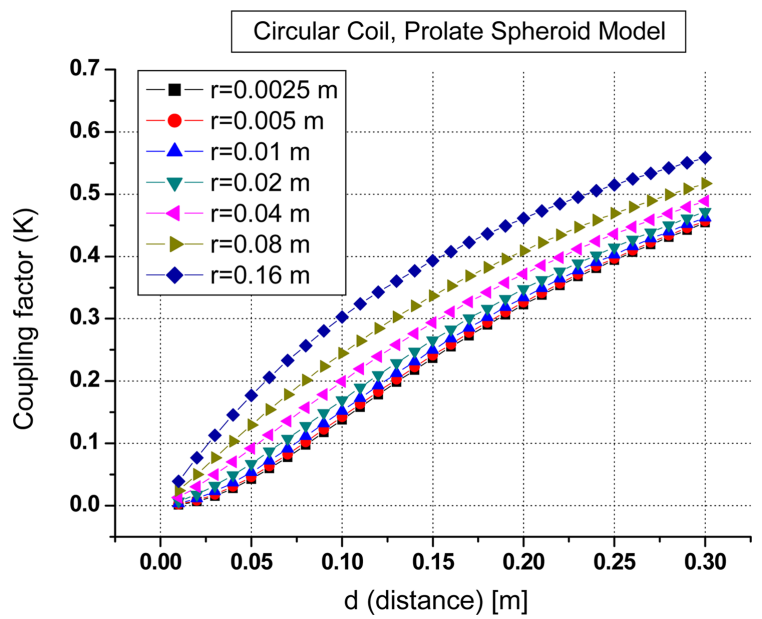

Fig. 9. (Color online) Coupling factor $K$ of circular coil according to coil radius $r$ and distance $d$ (prolate spheroid model).

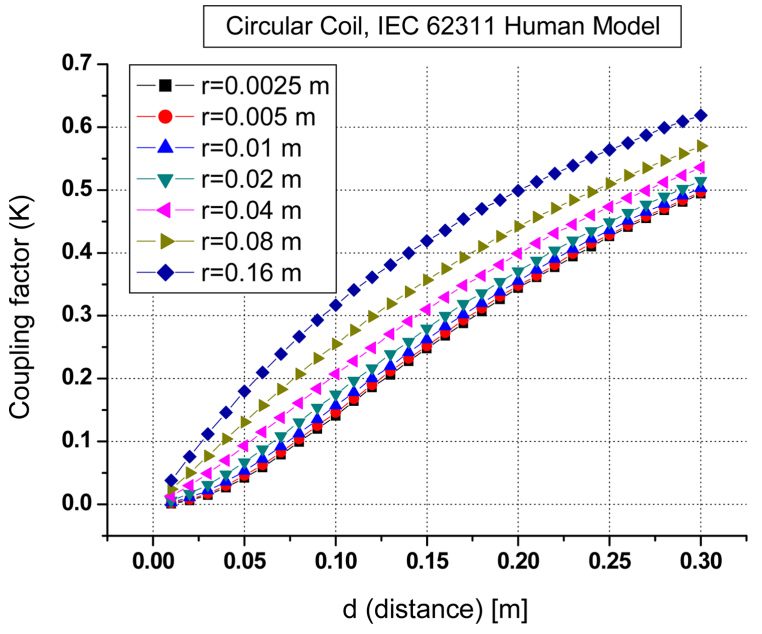

Fig. 10. (Color online) Coupling factor $K$ of circular coil according to coil radius $r$ and distance $d$ (IEC 62311 human model).

where $\sigma=0.2 \mathrm{~S} / \mathrm{m}$ is the conductivity of human model, $B$ $=1.25 \mu \mathrm{T}$ is magnitude of uniform flux density, and $r_{h}$ is radius of human model torso. For prolate spheroid, $r_{h}=$ $0.2 \mathrm{~m}$ and $J_{\text {uniform }}=7.85 \mu \mathrm{A} / \mathrm{m}$. For IEC 62311 human model, $r_{h}=0.175 \mathrm{~m}$ and $J_{\text {uniform }}=6.87 \mu \mathrm{A} / \mathrm{m}$.

Plots of coupling factors for different non-uniform sources and human models are shown in Figs. 9-12. In general, $K$ is increased as distance $d$ is increased. This trend agrees with the fact that the characteristics of the magnetic field from a localized source resemble those of the uniform field as the distance $d$ is increased. In addition, it can be observed that $K$ is also increased as radius $r$ of circular coil or gap $e$ of parallel wires is increased. For all cases considered (at $d=30 \mathrm{~cm}$ or less), $K$ values were less than 0.65 . At $d=20 \mathrm{~cm}$ or less, $K$ values were less than 0.55 , and at $d=10 \mathrm{~cm}$ or less, $K$

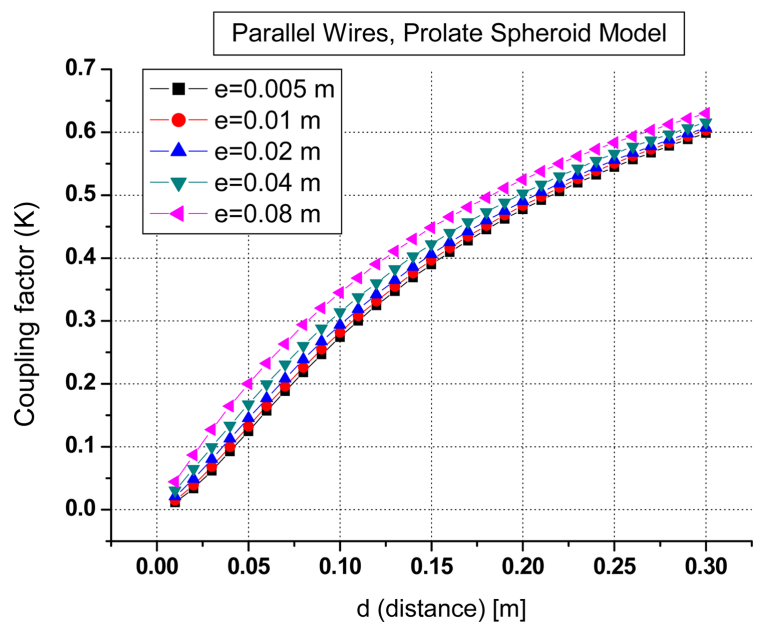

Fig. 11. (Color online) Coupling factor $K$ of parallel wires according to gap $e$ and distance $d$ (prolate spheroid model). 


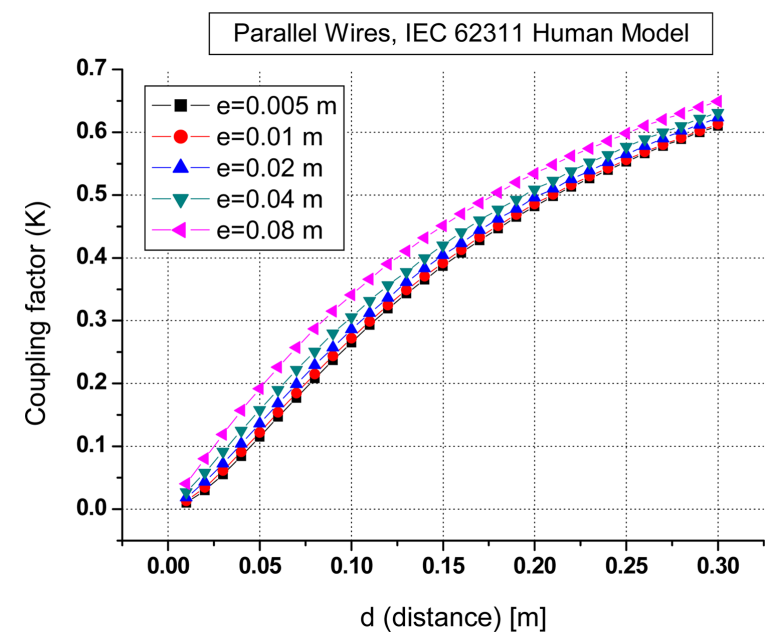

Fig. 12. (Color online) Coupling factor $K$ of parallel wires according to gap $e$ and distance $d$ (IEC 62311 human model).

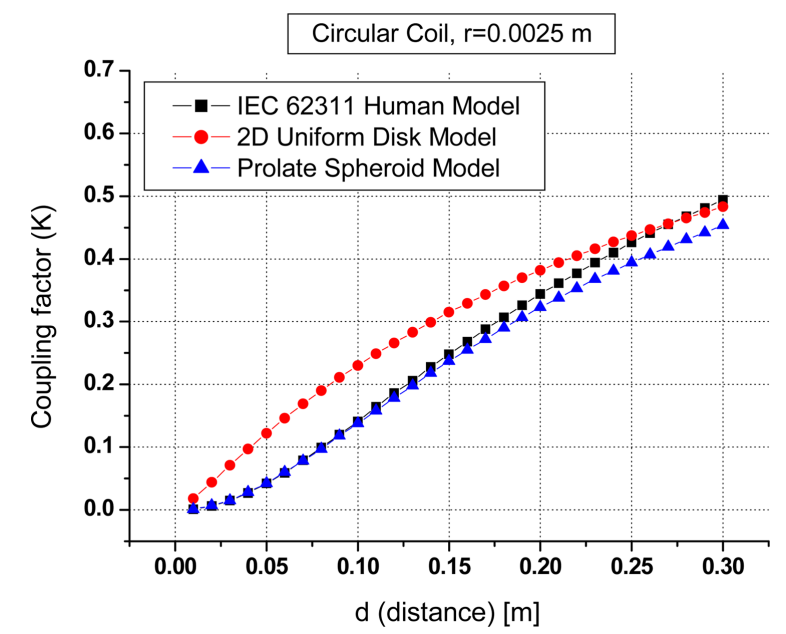

Fig. 13. (Color online) Coupling factor $K$ of circular coil obtained using different human models $(r=0.0025 \mathrm{~m})$.

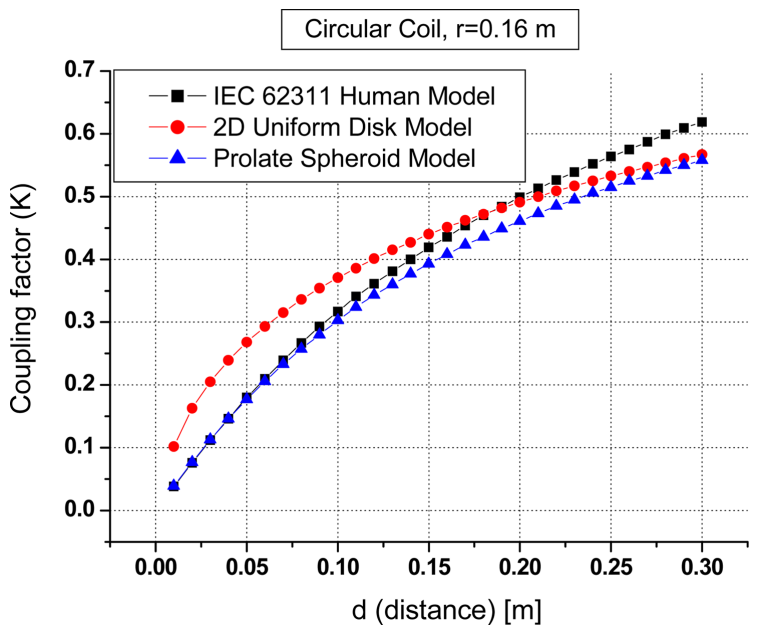

Fig. 14. (Color online) Coupling factor $K$ of circular coil obtained using different human models $(r=0.16 \mathrm{~m})$.

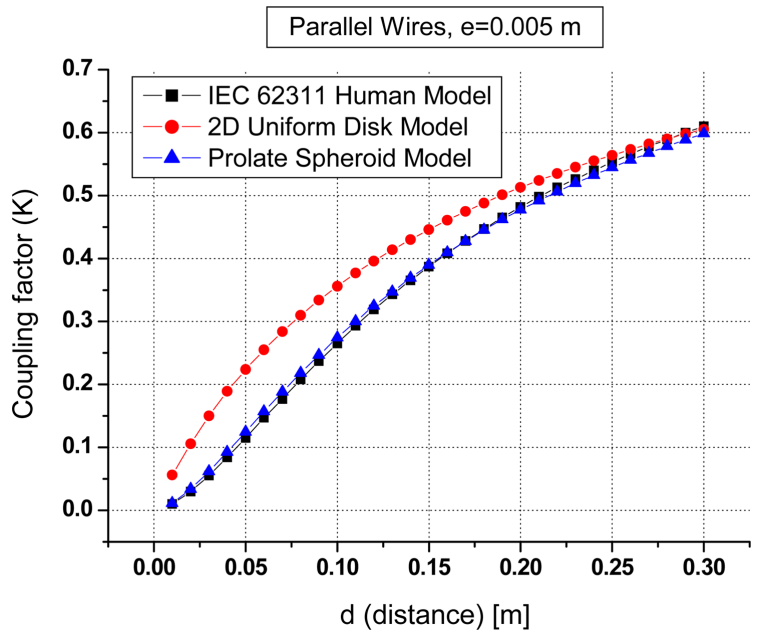

Fig. 15. (Color online) Coupling factor $K$ of parallel wires obtained using different human models $(e=0.005 \mathrm{~m})$.

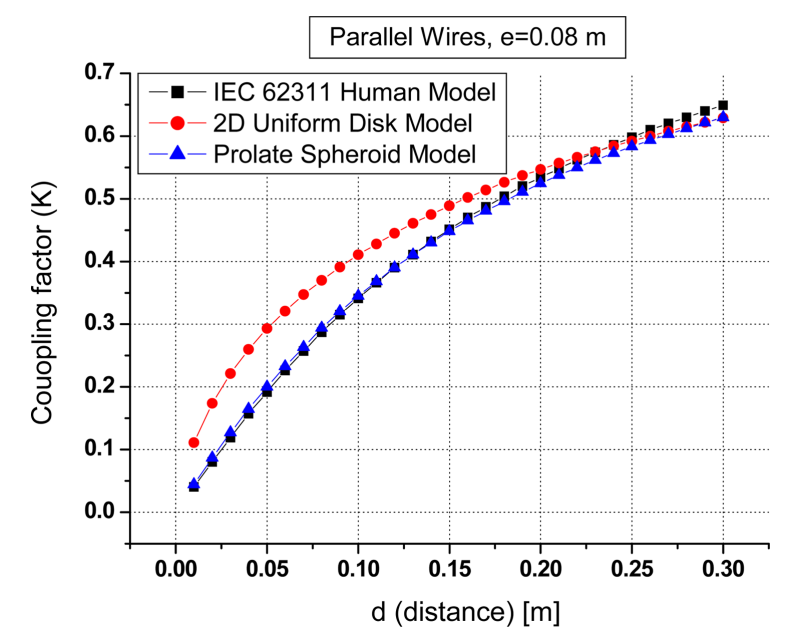

Fig. 16. (Color online) Coupling factor $K$ of parallel wires obtained using different human models $(e=0.08 \mathrm{~m})$.

values were less than 0.35 .

To analyze the dependence of coupling factor $K$ on the human model, $K$ is plotted for 3 different human models (uniform disk, prolate spheroid, and IEC 62311 model) when coil radius $r$ or wire gap $e$ is set as the same value (Figs. 13-16). The coupling factor of uniform disk is directly quoted from IEC 62226-2-1 standard [4].

Close inspection of Figs. 13-16 reveals that there exist considerable discrepancies in coupling factor between different human models (2D uniform disk model in particular). Using IEC 62311 human model as the reference case, the relative differences in $K$ values of 2D disk model and prolate spheroid are summarized for several values of distance $d$ in Tables 1-4. It can be seen that, in some cases, 2D uniform disk model has relative difference over $50 \%$. On the other hand, relative difference of prolate 
Table 1. Relative differences in $K$ values of circular coil $(r=0.0025 \mathrm{~m})$.

\begin{tabular}{cccccc}
\hline \hline Human model type & \multicolumn{5}{c}{ Relative difference in $K$ [\%] } \\
\cline { 2 - 6 } & $d=0.1 \mathrm{~m}$ & $d=0.15 \mathrm{~m}$ & $d=0.2 \mathrm{~m}$ & $d=0.25 \mathrm{~m}$ & $d=0.3 \mathrm{~m}$ \\
\hline 2D uniform disk model & 63.12 & 27.02 & 11.05 & 2.58 & 2.23 \\
Prolate spheroid model & 2.13 & 4.44 & 6.10 & 7.51 & 8.10 \\
\hline
\end{tabular}

Table 2. Relative differences in $K$ values of circular coil $(r=0.16 \mathrm{~m})$.

\begin{tabular}{cccccc}
\hline \hline Human model type & \multicolumn{4}{c}{ Relative difference in $K[\%]$} \\
\cline { 2 - 6 } & $d=0.1 \mathrm{~m}$ & $d=0.15 \mathrm{~m}$ & $d=0.2 \mathrm{~m}$ & $d=0.25 \mathrm{~m}$ & $d=0.3 \mathrm{~m}$ \\
\hline 2D uniform disk model & 17.03 & 5.01 & 1.60 & 5.50 & 8.40 \\
Prolate spheroid model & 4.42 & 6.21 & 7.62 & 8.69 & 9.85 \\
\hline
\end{tabular}

Table 3. Relative differences in $K$ values of parallel wires $(e=0.005 \mathrm{~m})$.

\begin{tabular}{cccccc}
\hline \hline Human model type & \multicolumn{5}{c}{ Relative difference in $K[\%]$} \\
\cline { 2 - 6 } & $d=0.1 \mathrm{~m}$ & $d=0.15 \mathrm{~m}$ & $d=0.2 \mathrm{~m}$ & $d=0.25 \mathrm{~m}$ & $d=0.3 \mathrm{~m}$ \\
\hline 2D uniform disk model & 34.34 & 15.25 & 6.43 & 1.99 & 0.82 \\
Prolate spheroid model & 3.53 & 0.69 & 0.93 & 1.52 & 1.89 \\
\hline
\end{tabular}

Table 4. Relative differences in $K$ values of parallel wires $(e=0.08 \mathrm{~m})$.

\begin{tabular}{cccccc}
\hline \hline \multirow{2}{*}{ Human model type } & \multicolumn{5}{c}{ Relative difference in $K[\%]$} \\
\cline { 2 - 6 } & $d=0.1 \mathrm{~m}$ & $d=0.15 \mathrm{~m}$ & $d=0.2 \mathrm{~m}$ & $d=0.25 \mathrm{~m}$ & $d=0.3 \mathrm{~m}$ \\
\hline 2D uniform disk model & 20.53 & 8.43 & 2.43 & 1.00 & 3.08 \\
Prolate spheroid model & 1.17 & 0.62 & 1.80 & 2.50 & 2.95 \\
\hline
\end{tabular}

spheroid model is in 0.6-10\% range. In addition, circular coil shows bigger discrepancies in $K$ values between different human models than those of parallel wires for most cases considered. These results suggest that $K$ values obtained using 2D uniform disk model such as in IEC 62226-2-1 standard can include considerable error, and $3 \mathrm{D}$ human models should be used instead for calculation of coupling factor.

\section{Conclusion}

In this paper, coupling factors were calculated in order to evaluate various non-uniform, low-frequency magnetic field exposure situations from localized sources. Two types of localized sources were considered; circular coil and parallel wires with balanced currents. For equivalent human models, prolate spheroid model and simplified human model from IEC 62311 standard were used. For all cases considered, coupling factor $K$ values were less than 0.65 . The calculated coupling factor values were compared with those from IEC 62226-2-1 standard which were computed using 2D uniform disk model. The calculation results show that there are considerable discrepancies between coupling factor values obtained using $2 \mathrm{D}$ uniform disk model and 3D models. The relative difference is more than $50 \%$ in some cases. These results suggest that $3 \mathrm{D}$ equivalent human models should be used for coupling factor calculation. It is expected that coupling factors calculated in this paper can be used to estimate induced current density in human body under practical lowfrequency magnetic field exposure situations, and can facilitate the procedures for determining compliance with EMF safety guidelines. To further improve the accuracy of coupling factor calculations, adoption of anatomical human models with inhomogeneous biological tissue composition can be considered for future works.

\section{Acknowledgment}

This work was supported by the IT R\&D program of MSIP/IITP [B0138-16-1002, Study on the EMF exposure control in smart society]. 


\section{References}

[1] International Commission on Non-Ionizing Radiation Protection, Health Physics 74, 494 (1998).

[2] International Commission on Non-Ionizing Radiation Protection, Health Physics 99, 818 (2010).
[3] IEEE Standard C95.1-2005, IEEE, New York (2006) pp. 14-15.

[4] International Standard IEC 62226-2-1, IEC, Geneva (2004).

[5] International Standard IEC 62311, IEC, Geneva (2007).

[6] COMSOL Inc.(www.comcol.com).

[7] Y.-M. Park, H.-J. Song, and J.-K. Byun, J. Magn. 19, 174 (2014). 